\title{
Follow-up Search for UHE Photons from Gravitational Wave Sources with the Pierre Auger Observatory
}

\author{
Philip Ruehl ${ }^{a, *}$ on behalf of the Pierre Auger $^{b}$ Collaboration \\ (a complete list of authors can be found at the end of the proceedings) \\ ${ }^{a}$ University of Siegen, Walter-Flex-Str. 3, Siegen, Germany \\ ${ }^{b}$ Observatorio Pierre Auger, Av. San Martín Norte 304, 5613 Malargüe, Argentina \\ E-mail: spokespersons@auger.org
}

\begin{abstract}
Multimessenger astronomy has become increasingly important during the past decade. Some astronomical objects have already been successfully observed in the light of multiple messenger signals, allowing for a much deeper understanding of their physical properties. The Pierre Auger Observatory has taken part in multimessenger astronomy with an exhaustive exploration of the ultra-high-energy sky. In this contribution, for the first time, a search for UHE photons from the sources of gravitational waves is presented. Interactions with the cosmic background radiation fields are expected to attenuate any possible flux of ultra-high-energy photons from distant sources and a non-negligible background of air shower events with hadronic origin makes an unambiguous identification of primary photons a challenging task. In the analysis presented here, a selection strategy is applied to both GW sources and air shower events aiming to provide maximum sensitivity to a possible photon signal. At the same time, a window is kept open for hypothetical new-physics processes, which might allow for much larger interaction lengths of photons in the extragalactic medium. Preliminary results on the UHE photon fluence from a selection of GW sources, including the binary neutron star merger GW170817 are presented.
\end{abstract}

$37^{\text {th }}$ International Cosmic Ray Conference (ICRC 2021)

July 12th - 23rd, 2021

Online - Berlin, Germany

\footnotetext{
${ }^{*}$ Presenter
} 


\section{Introduction}

Since the first detection of gravitational waves (GWs) with the advanced LIGO and Virgo Observatories in 2015, GW observations have become a key element for multimessenger astronomy. In the past years, the transient sources of these GWs have been analyzed by various astronomical instruments throughout the accessible part of the electromagnetic spectrum and in the light of high-energy neutrinos. See [1] for a description.

With its unique exposure to ultra-high-energy (UHE) radiation, the Pierre Auger Observatory has joined the global campaign in the multimessenger astronomy of GW sources by searching for UHE neutrinos [2-4]. Here, we extend these follow-up observations to UHE photons beyond $10 \mathrm{EeV}$ in temporal coincidence with GW observations from observation runs O1, O2 and O3a. Despite UHE photons having a limited mean free path due to their interactions with the cosmic background radiation fields, we show that a dedicated search in a multimessenger approach can provide new information on GW sources. To maintain a maximum susceptibility to the still sparse number of GW observations, the surface detecor (SD) array of the Pierre Auger Observatory is used here. The SD array comprises more than 1600 water Cherenkov detectors on a triangular grid with a spacing of $1.5 \mathrm{~km}$. Thanks to its design, the SD has a duty cycle of almost $100 \%$ [5].

\section{Search for UHE Photons}

The bulk of data received at the Pierre Auger Observatory originates in cosmic rays of hadronic nature [6]. The identification of a possible component of UHE photons among the diffuse flux of cosmic rays with the Pierre Auger Observatory has been discussed in [7, 8]. No statistically significant excess of primary UHE photon candidate events has been found therein and, hence, stringent upper limits on the diffuse photon flux above $2 \times 10^{17} \mathrm{eV}$ could be derived. A dedicated analysis studying possible steady point sources of UHE photons has also been published [10, 11]. Again, no excess of photon-like events could be found neither in a blind search on the whole sky nor in a targeted search from a set of selected source candidates.

To identify primary photons from transient point sources among the background of hadronic cosmic rays, the photon-discrimination method from [8] has been adopted in this work. This method utilizes the data recorded by the SD and selects photon candidate events based on the signal distribution in the plane perpendicular to the shower axis and the shapes of the signal time traces measured by the water Cherenkov SD stations. Two observables are used, $L_{\mathrm{LDF}}$ and $\Delta$, respectively related to the lateral distribution function (LDF) and to the rise time of the signal traces. They are optimized for air showers with incident zenith angles $\theta$ between $30^{\circ}$ and $60^{\circ}$ and photon energies $E_{\gamma}$ between $10^{19.0} \mathrm{eV}$ and $10^{20.5} \mathrm{eV}$. Since the standard energy reconstruction of the Auger SD uses data events for the calibration of the absolute energy scale, the energy of a possible photon-induced air shower would be underestimated due to its reduced hadronic component. To obtain a less biased estimator for the photon energy $E_{\gamma}$, an alternative energy reconstruction method has been used in [8]. This method is based on an iterative procedure which recursively uses the elongation rate of photon-induced air showers and the universal profile of the electromagnetic shower component to obtain a series of photon energy estimators which converges to $E_{\gamma}[9]$. 


\section{Exposure}

To discard low-quality events, a number of selection criteria has been imposed on the data. Events are required to have 6 active SD stations around the station with the highest signal ("active hexagon") and have successfully reconstructed shower axis, LDF and photon energy. For calculating the discriminating air shower observables $\Delta$ and $L_{\mathrm{LDF}}$, an additional event selection has to be applied. Events with reconstructed hadronic energy ${ }^{1} E_{\mathrm{hd}}<10^{18} \mathrm{eV}$ are rejected. Events which do not have a triggered station more than $1000 \mathrm{~m}$ away from the shower axis (excluding stations with a saturated low-gain channel) are also rejected to ensure a proper reconstruction of $L_{\mathrm{LDF}}$. Events are also required to have a reconstructed shower maximum $X_{\max }{ }^{2}$ which is not more than $50 \mathrm{~g} \mathrm{~cm}^{-2}$ below ground. These restrictions limit the photon detection efficiency depending on energy and zenith angle of the primary particle. Assuming an $E_{\gamma}^{-2}$ energy spectrum, the zenith-angle-averaged photon efficiency in the energy range $10^{19.0} \mathrm{eV} \leq E_{\gamma}<10^{20.5} \mathrm{eV}$ is found to be $\epsilon=0.483 \pm 0.008$ using CORSIKA [13] simulations. With the photon detection efficiency given as a function of energy and direction, the exposure to UHE photons from a transient point source at zenith angle $\theta_{\mathrm{GW}}$ during the obervation period $\Delta t=t_{1}-t_{0}$ is given by

$$
\mathcal{E}\left(E_{\gamma}, \theta_{\mathrm{GW}}, \Delta t\right)=\int_{t_{0}}^{t_{1}} \mathrm{~d} t A(t) \epsilon\left(E_{\gamma}, \theta_{\mathrm{GW}}(t)\right) \Theta_{\mathrm{FoV}}\left(\theta_{\mathrm{GW}}(t)\right) \cos \left(\theta_{\mathrm{GW}}(t)\right)
$$

with $A(t)$ being the time-dependent aperture of the Auger SD array as determined by the number of active hexagons at a given moment. The step-function $\Theta_{\mathrm{FoV}}$ accounts for the fraction of the observation time in which the zenith angle $\theta_{\mathrm{GW}}$ of the GW source is between $30^{\circ}$ and $60^{\circ}$.

For the search period, two mutual exclusive time windows of $\Delta t_{\text {short }}=1000 \mathrm{~s}$ starting at $t_{0}=-500 \mathrm{~s}$ before the GW event time and $\Delta t_{\text {long }}=1$ day starting at $t_{0}=+500 \mathrm{~s}$ after the GW event time have been chosen as in with [2,4]. After spectral integration, the spectrum-weighted exposure is a function of both right ascension and declination of the source for a $1000 \mathrm{~s}$ time window. Integration over a sidereal day in practive makes the spectrum-weighted exposure only dependent on declination as shown in Fig. 1.

\section{Gravitational Wave Event Selection}

To keep the sensitivity to a possible photon signal as high as possible, GW events are carefully selected by their localization quality and distance. Close and well localized sources are preferred over distant and poorly localized ones. Hence, optimal results can be obtained while keeping the expected amount of background at a managable level.

The localization of a GW source is communicated by the LIGO/Virgo Collaborations via a probability density distribution on the sky (sky localization map). An analysis of these distributions leads to the conclusion that using their 50\% contour as the search region in the sky is a reasonable compromise between the expected amount of background (which is proportional to the solid angle

\footnotetext{
${ }^{1}$ energy estimator obtained by the standard SD energy reconstruction [12].

${ }^{2}$ The shower maximum is not directly accessible with the SD. An alternative estimator for $X_{\max }$ is obtained within the iterative process of reconstructing the photon energy $E_{\gamma}$ which is explained in [9].
} 


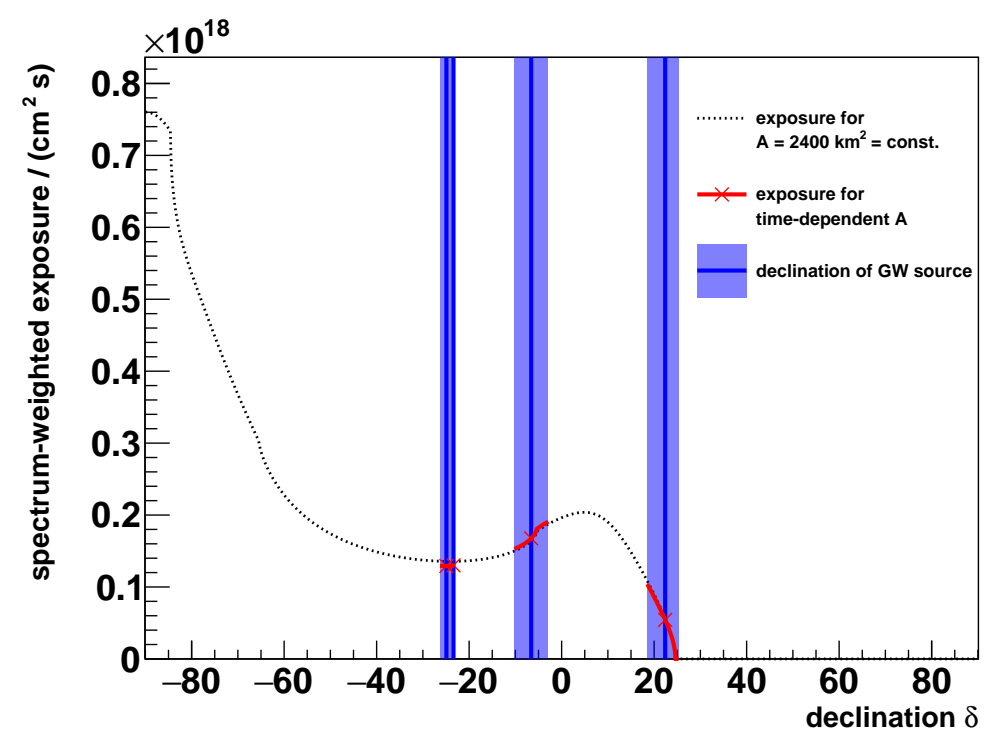

Figure 1: The spectrum-weighted exposure during a full sidereal day for a benchmark value of a typical aperture of $2.4 \times 10^{13} \mathrm{~cm}^{2}$. The blue bars indicate the declination bands that are covered by the four selected GW events (see Sec. 4) with the most likely direction marked by the dark line (GW event-IDs from left to right: GW190814, GW170817, GW190701_203306, GW170818). The indication of a declination band has been omitted for GW170817 since the host galaxy of this event has been identified and, hence, the uncertainty of the source direction is negligible. The red solid lines display the actual exposure to photons from these GW events taking into account time dependent fluctuations of the aperture.

of the analyzed sky region) and the confidence level at which the true source is localized within the search region. To also take into account the directional resolution of the Auger SD of about $1^{\circ}$ for photon-induced air showers above $10^{19} \mathrm{eV}^{3}$, the sky localization maps of GW sources are convolved with a corresponding Gaussian distribution before deriving the 50\% contour.

Three classes of GW events have been defined for which the 50\% localization region will be analyzed for coincident photon candidate events. The first class (class 1) comprises GW sources with a maximum contour size of $100 \mathrm{deg}^{2}$ which are required to not be farther away than $140 \mathrm{Mpc}$ mean value of the distance resolution. The maximum contour size of $100 \mathrm{deg}^{2}$ is chosen such that the significance of a photon candidate event within a $1000 \mathrm{~s}$ time window would in any case be above the $5 \sigma$ level. The maximum distance of $140 \mathrm{Mpc}$ is chosen such as to reject $\mathrm{GW}$ sources from which no photons are expected to reach the Earth even under the most optimistic assumptions about the photon luminosity of the source and its emission pattern and spectrum. For this choice, a "photon horizon" $h_{\gamma}$ has been estimated. This photon horizon is the distance beyond which the photon luminosity of even the brightest GW source so far, i.e. GW190521 with total energy radiated in GW of $7.6_{-1.9}^{+2.2} \mathrm{M}_{\odot}$ [15], could not possibly be constrained to be less than its total luminosity. Using CRPropa 3 [16] to simulate the propagation and attenuation of a flux of UHE photons in the extragalactic medium, a maximum attenuation length of $7 \mathrm{Mpc}$ has been found for photons at $100 \mathrm{EeV}$. This leads to a maximum photon horizon of $h_{\gamma}^{\text {iso }} \sim 90 \mathrm{Mpc}$. Since this

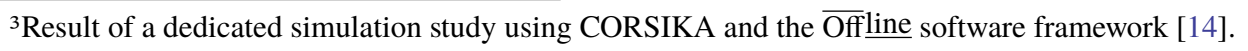


horizon holds for isotropic emission, it might underestimate the directional luminosity of sources with strongly beamed jets. To account also for sources which might expose narrow jets with halfopening angles $\lesssim 3^{\circ}$ (c.f. GW170817 [17]) pointing directly towards Earth, only sources with $D_{\mathrm{L}}>h_{\gamma} \sim 140 \mathrm{Mpc}$ are rejected. For those sources, the exponential attenuation of UHE photons in the extragalactic medium in any case overweights the enhancement of any possible photon flux along the beam axis. A second class (class 2) of accepted GW events is defined which allows especially close sources to be analyzed up to a maximum allowed contour size of $720 \mathrm{deg}^{2}$. For such sources with luminosity distance $D_{\mathrm{L}} \leq 40 \mathrm{Mpc}$ (which is the distance of the closest source of GWs observed so far, the binary neutron star (BNS) merger GW170817), there is a good chance to actually observe a potential UHE photon flux or place strong physical constraints. Finally, to keep a window open for potentially unexpected discoveries, a third subset (class 3) of especially well localized sources is introduced. These sources are characterized by a $50 \%$ contour smaller than $20 \mathrm{deg}^{2}$ and accepted independent of their distance. From such a small region in the sky, the expected background would be negligible (c.f. Sec. 6) and the detection of a coincident photon candidate event would be a strong hint towards new physics capable of suppressing the interactions of UHE photons with the cosmic background radiation fields. A possible new-physics process that could give rise such an effect is the oscillation between UHE photons and axions [18]. The selection criteria can be summarized as

$$
\begin{array}{lll}
\left(D_{\mathrm{L}}<140 \mathrm{Mpc}\right. & \text { and } \left.\quad \Omega_{50 \%}<100 \mathrm{deg}^{2}\right) & \text { "class 1" } \\
\left(D_{\mathrm{L}}<40 \mathrm{Mpc}\right. & \text { and } \left.\quad \Omega_{50 \%}<720 \mathrm{deg}^{2}\right) & \text { "class 2" } \\
\left(D_{\mathrm{L}}<\infty\right. & \text { and } \left.\quad \Omega_{50 \%}<20 \mathrm{deg}^{2}\right) & \text { "class 3". }
\end{array}
$$

In Fig. 2, the accepted regions in the space of source localization $\Omega_{50 \%}$ and luminosity distance $D_{\mathrm{L}}$ are visualized on top of the distribution of all confident GW observations published in GWTC-1 [19] and GWTC-2 [20].

\section{Preliminary Results}

In total, five GW events pass the event selection described above. Four of those, namely GW170817, GW170818, GW190701_203306 and GW190814, had some overlap with the field of view of the SD during the 1 day time window. These events belong to different source classes which are a BNS merger (GW170817) [22], two binary black hole mergers (GW170818, GW190701_203306) [19,20] and a black hole-neutron star merger candidate (GW190814) [23]. The BNS event GW170817 poses a special case since its host galaxy has been identified through electromagnetic follow-up observations as NGC 4993 [22] providing a much better localization than through the GW observation alone. Instead of using the 50\% contour of the convolved sky map, in this case, a circular region with angular radius of $2^{\circ}$ has been chosen as the search region in compliance with the analysis of the blazar TXS 0506+056 in [24]. This region is chosen such that $90 \%$ of all UHE photons reaching the SD from NGC4993 are expected to be reconstructed within that region. 


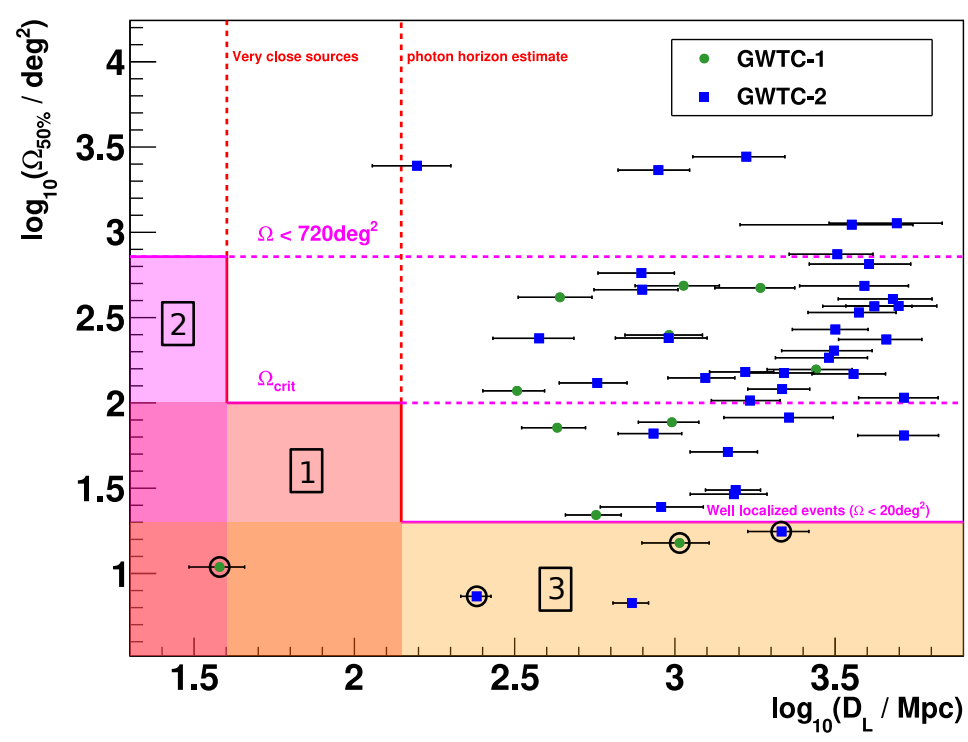

Figure 2: All GW events from GWTC-1 (green dots) and GWTC-2 (blue squares) in the space of source distance $D_{L}$ and localization $\Omega_{50 \%}$. The shaded regions define the set of accepted events according to the selection criteria described in the text while the numbers refer to the three different source classes in the acceptance region. The localization region of the events, that are marked by a black circle also have an overlap with the field of view of the SD.

All four GW events have been analyzed with respect to a possible UHE photon signal. No coincident air shower events have been found in the data of the Pierre Auger Observatoy and, hence, no coincident photon candidates could be identified. As a consequence, upper limits on the spectral fluence

$$
\mathcal{F}:=\int_{t_{0}}^{t_{1}} \int_{E_{0}}^{E_{1}} \mathrm{~d} t \mathrm{~d} E_{\gamma} \frac{\mathrm{d} \Phi_{\gamma}^{\mathrm{GW}}}{\mathrm{d} E_{\gamma}} E_{\gamma}
$$

of UHE photons have been placed at $90 \% \mathrm{CL}$ for each GW source. Here, $\Phi_{\gamma}^{\mathrm{GW}}$ represents the UHE photon flux from a GW point source. The upper limits $\mathcal{F}^{U L}$ on the spectral fluence inherit the declination dependence of the exposure. Therefore, the variation of $\mathcal{F}^{U L}$ due to the declination band covered by the search region differs for each event. It is indicated in Fig. 3 by the blue bars. This uncertainty has been omitted for GW170817 since its source NGC 4993 is very well localized in the sky. On top of it there is the uncertainty due to the variation of the spectral index $\alpha \in[-2.3,-1.7]$ that has been considered which imposes an additional uncertainty of order $20 \%$, indicated in Fig. 3 by the red bars.

\section{Sensitivity}

The photon-discrimination method described above has a non-negligible rate of false-positive detections. In [8], out of all air shower events recorded during the full operating period of $14.5 \mathrm{yr}$, eleven events passed the photon candidate selection and were found to be consistent with the 


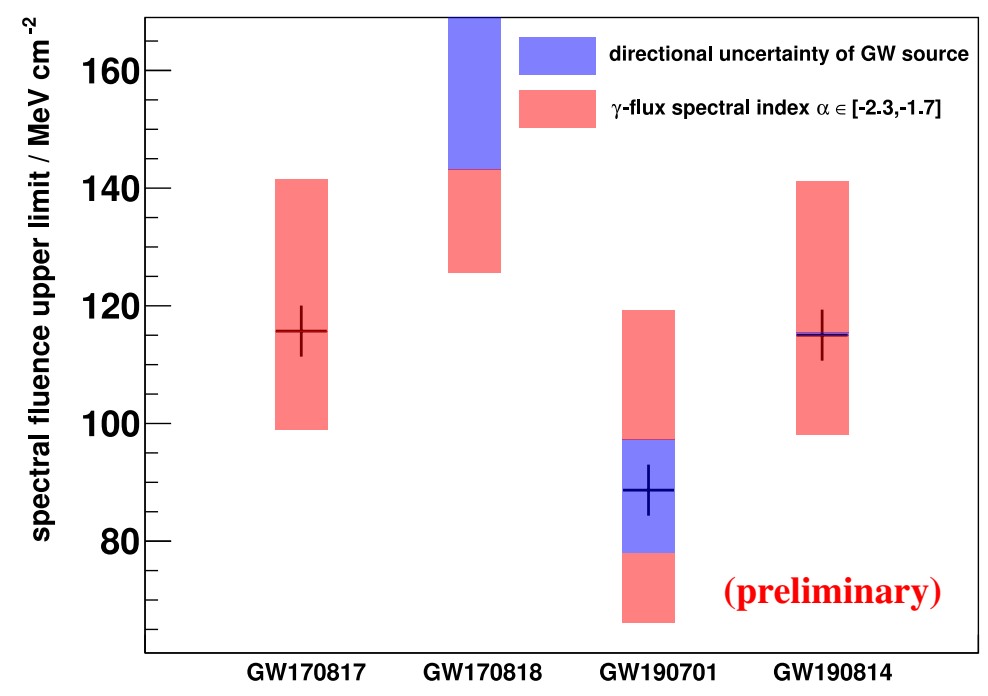

Figure 3: Preliminary upper limits $(90 \%$ CL) on the spectral fluence for the four GW sources that pass the selection criteria defined Sec. 4. Uncertainties imposed by the limited sky localization of the sources as well as a variation of the spectral index $\alpha$ are indicated by the blue and red bars respectively. Due to the sky localization of GW170818 close to the edge of the field of view, the central value of $\mathcal{F}^{U L}(\mathrm{GW} 170818)=$ $270 \mathrm{MeVcm}^{-2}$ is not displayed within the axis range. The uncertainty band of this event has no upper bound since the localization region is not fully covered by the field of view.

hypothesis of hadronic background events. This number may serve as an estimate for the background rate associated to the present analysis. Given an amount of expected background, the sensitivity to a possible signal of primary photons can be quantified through the confidence level at which the background hypothesis can be rejected in the case of a single photon candidate detection in coincidence with a GW event. For a given number of observed photon candidate events and a given amount of background events, a confidence interval for the true number of photons can be obtained through the construction described by Feldman and Cousins (FC) [21]. Depending on the confidence level (CL), the lower limit of these interval may or may not be equal to zero. Thus, as a convenient measure for the sensitivity, we define the photon significance as the highest confidence level at which the FC lower limit is not consistent with zero for the given background and an assumed single photon candidate event. Assuming a single photon candidate event within any of the four sky regions and time windows analyzed here, the expected amount of background events, which is $b=3.2 \times 10^{-6}$, leads to a photon significance of $4.65 \sigma$, which means that the hypothesis of such an event originating in the background could be rejected at $\mathrm{CL}$ of $4.65 \sigma$. For each single $\mathrm{GW}$ event the significance would be at a level of $4.95 \sigma\left(b=7.1 \times 10^{-7}\right)$ for GW170817, $5.0 \sigma\left(b=4.6 \times 10^{-7}\right)$ for GW170818, $4.8 \sigma\left(b=1.6 \times 10^{-6}\right)$ for GW190701_203306 and $5.0 \sigma\left(b=4.9 \times 10^{-7}\right)$ for GW190814. 


\section{Summary}

In this contribution, a first approach to constrain UHE photons from different GW sources using the SD array of the Pierre Auger Observatory has been presented. For this purpose a dedicated selection strategy for both GW sources and air shower events has been developed which aims to keep the sensitivity towards a possible photon signal at a high level despite an irreducible background of hadronic primaries. With this selection strategy a detection at a significance beyond the $5 \sigma$ level is possible. Out of the GW catalogs GWTC-1 and GWTC-2, which comprise 50 confident GW detections in total, four GW events - including the binary neutron star merger GW170817 and the black hole-neutron star merger candidate GW190814 - were selected following this strategy and analyzed for UHE photons. No photon candidate events have been found and preliminary upper limits on the spectral fluence of UHE photons were shown here for the first time.

\section{References}

[1] M. Branchesi, J. Phys. Conf. Ser. 718 (2016) 22004.

[2] A. Aab et al., Phys. Rev. D 94 (2016) 122007 [1608.07378].

[3] A. Albert et al., Astrophys. J. Lett. 850 (2017) L35 [1710.05839].

[4] M. Schimp [Pierre Auger Coll.], these proceedings.

[5] A. Aab et al., Nucl. Instrum. Meth. A 798 (2015) 172 [1502.01323].

[6] A. Aab et al., J. Cosmol. Astropart. P. 04 (2017) 038 [1612 . 07155].

[7] A. Aab et al., J. Cosmol. Astropart. P. 04 (2017) 09 [1612.01517].

[8] J. Rautenberg [Pierre Auger Coll.], Proc. 36th Int. Cosmic Ray Conf., Madison, USA (2019) PoS(ICRC2019)398 [1909.09073].

[9] P. Billoir et al., [astro-ph/0701583] (2007).

[10] A. Aab et al., Astrophys. J. Lett. 837 (2016) L25 [1612.04155].

[11] A. Aab et al., Astrophys. J. 789 (2014) 160 [1406.2912].

[12] V. Verzi [Pierre Auger Coll.], Proc. 36th Int. Cosmic Ray Conf., Madison, USA (2019) PoS(ICRC2019)459 [1909.09073].

[13] D. Heck et al., Report FZKA (1998) 6019.

[14] S. Argirò et al., Nucl. Instrum. Meth. A 580 (2007) 1485 [0707. 1652].

[15] R. Abbott et al., Astrophys. J. Lett. 900 (2020) L13 [2009 . 01190].

[16] R. Alves Batista et al., J. Cosmol. Astropart. P. 05 (2016) 38 [1603.07142].

[17] K. P. Mooley et al., Astrophys. J. Lett. 868 (2018) L11 [1810.12927].

[18] M. Fairbairn et al., Phys. Rev. D 84 (2011) 125019 [0901.4085].

[19] B. P. Abbott et al., Phys. Rev. X 9 (2019) 031040 [1811. 12907].

[20] R. Abbott et al., Phys. Rev. X 11 (2021) 021053 [2010 . 14527].

[21] G. J. Feldman, R. D. Cousins, Phys. Rev. D 57 (1998) 3873 [9711021].

[22] B. P. Abbott et al., Astrophys. J. Lett. 848 (2017) L13 [1710. 05834].

[23] R. Abbott et al., Astrophys. J. Lett. 896 (2020) L44 [2006 . 12611].

[24] A. Aab et al., Astrophys. J. 902 (2020) 105 [2010. 10953]. 


\section{The Pierre Auger Collaboration}

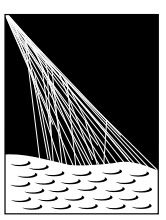

PIERRE

AUSGERVATORY

P. Abreu ${ }^{72}$, M. Aglietta ${ }^{54,52}$, J.M. Albury ${ }^{13}$, I. Allekotte ${ }^{1}$, A. Almela ${ }^{8,12}$, J. Alvarez-Muñiz ${ }^{79}$, R. Alves Batista ${ }^{80}$, G.A. Anastasi ${ }^{63,52}$, L. Anchordoqui ${ }^{87}$, B. Andrada ${ }^{8}$, S. Andringa ${ }^{72}$, C. $A r a m 0^{50}$, P.R. Araújo Ferreira ${ }^{42}$, J. C. Arteaga Velázquez ${ }^{67}$, H. Asorey ${ }^{8}$, P. Assis ${ }^{72}$, G. Avila ${ }^{11}$, A.M. Badescu ${ }^{75}$, A. Bakalova ${ }^{32}$, A. Balaceanu ${ }^{73}$, F. Barbato ${ }^{45,46}$, R.J. Barreira $\mathrm{Luz}^{72}$, K.H. Becker ${ }^{38}$, J.A. Bellido ${ }^{13,69}$, C. Berat ${ }^{36}$, M.E. Bertaina ${ }^{63,52}$, X. Bertou ${ }^{1}$, P.L. Biermann ${ }^{b}$, V. Binet ${ }^{6}$, K. Bismark ${ }^{39,8}$, T. Bister ${ }^{42}$, J. Biteau ${ }^{37}$, J. Blazek ${ }^{32}$, C. Bleve ${ }^{36}$, M. Boháčová ${ }^{32}$, D. Boncioli ${ }^{57,46}$, C. Bonifazi ${ }^{9,26}$, L. Bonneau Arbeletche ${ }^{21}$, N. Borodai ${ }^{70}$, A.M. Botti ${ }^{8}$, J. Brack ${ }^{d}$, T. Bretz ${ }^{42}$, P.G. Brichetto Orchera ${ }^{8}$, F.L. Briechle ${ }^{42}$, P. Buchholz ${ }^{44}$, A. Bueno ${ }^{78}$, S. Buitink ${ }^{15}$, M. Buscemi ${ }^{47}$, M. Büsken ${ }^{39,8}$, K.S. Caballero-Mora ${ }^{66}$, L. Caccianiga ${ }^{59,49}$, F. Canfora ${ }^{80,81}$, I. Caracas ${ }^{38}$, J.M. Carceller ${ }^{78}$, R. Caruso ${ }^{58,47}$, A. Castellina ${ }^{54,52}$, F. Catalani ${ }^{19}$, G. Cataldi ${ }^{48}$, L. Cazon ${ }^{72}$, M. Cerda ${ }^{10}$, J.A. Chinellato ${ }^{22}$, J. Chudoba ${ }^{32}$, L. Chytka ${ }^{33}$, R.W. Clay ${ }^{13}$, A.C. Cobos Cerutti ${ }^{7}$, R. Colalillo ${ }^{60,50}$, A. Coleman ${ }^{93}$, M.R. Coluccia ${ }^{48}$, R. Conceição ${ }^{72}$, A. Condorellii ${ }^{45,46}$, G. Consolati ${ }^{49,55}$, F. Contreras ${ }^{11}$, F. Convenga ${ }^{56,48}$, D. Correia dos $\operatorname{Santos}^{28}$, C.E. Covault ${ }^{85}$, S. Dasso ${ }^{5,3}$, K. Daumiller ${ }^{41}$, B.R. Dawson ${ }^{13}$, J.A. Day ${ }^{13}$, R.M. de Almeida ${ }^{28}$, J. de Jesús ${ }^{8,41}$, S.J. de Jong ${ }^{80,81}$, G. De Mauro ${ }^{80,81}$, J.R.T. de Mello Neto ${ }^{26,27}$, I. De Mitri ${ }^{45,46}$, J. de Oliveira ${ }^{18}$, D. de Oliveira Franco ${ }^{22}$, F. de Palma ${ }^{56,48}$, V. de Souza ${ }^{20}$, E. De Vito ${ }^{56,48}$, M. del Río ${ }^{11}$, O. Deligny ${ }^{34}$, L. Deval ${ }^{41,8}$, A. di Matteo ${ }^{52}$, C. Dobrigkeit ${ }^{22}$, J.C. D’Olivo ${ }^{68}$, L.M. Domingues Mendes ${ }^{72}$, R.C. $\operatorname{dos} \mathrm{Anjos}^{25}$, D. dos $\operatorname{Santos}^{28}$, M.T. Dova ${ }^{4}$, J. Ebr ${ }^{32}$, R. Engel ${ }^{39,41}$, I. Epicoco ${ }^{56,48}$, M. Erdmann ${ }^{42}$, C.O. Escobar ${ }^{a}$, A. Etchegoyen ${ }^{8,12}$, H. Falcke ${ }^{80,82,81}$, J. Farmer ${ }^{92}$, G. Farrar ${ }^{90}$, A.C. Fauth ${ }^{22}$, N. Fazzini ${ }^{a}$, F. Feldbusch ${ }^{40}$, F. Fenu ${ }^{54,52}$, B. Fick ${ }^{89}$, J.M. Figueira ${ }^{8}$, A. Filipčičč ${ }^{77,76}$, T. Fitoussi ${ }^{41}$, T. Fodran ${ }^{80}$, M.M. Freire ${ }^{6}$, T. Fujii ${ }^{92, e}$, A. Fuster ${ }^{8,12}$, C. Galea ${ }^{80}$, C. Galelli ${ }^{59,49}$, B. García ${ }^{7}$, A.L. Garcia Vegas ${ }^{42}$, H. Gemmeke ${ }^{40}$, F. Gesualdi ${ }^{8,41}$, A. Gherghel-Lascu ${ }^{73}$, P.L. Ghia ${ }^{34}$, U. Giaccari ${ }^{80}$, M. Giammarchi ${ }^{49}$, J. Glombitza ${ }^{42}$, F. Gobbi ${ }^{10}$, F. Gollan ${ }^{8}$, G. Golup ${ }^{1}$, M. Gómez Berisso $^{1}$, P.F. Gómez Vitale ${ }^{11}$, J.P. Gongora ${ }^{11}$, J.M. González ${ }^{1}$, N. González ${ }^{14}$, I. Goos ${ }^{1,41}$, D. Góra ${ }^{70}$, A. Gorgi ${ }^{54,52}$, M. Gottowik ${ }^{38}$, T.D. Grubb ${ }^{13}$, F. Guarino ${ }^{60,50}$, G.P. Guedes ${ }^{23}$, E. Guido ${ }^{52,63}$, S. Hahn $^{41,8}$, P. Hamal ${ }^{32}$, M.R. Hampel ${ }^{8}$, P. Hansen ${ }^{4}$, D. Harari ${ }^{1}$, V.M. Harvey ${ }^{13}$, A. Haungs ${ }^{41}$, T. Hebbeker ${ }^{42}$, D. Heck ${ }^{41}$, G.C. Hill ${ }^{13}$, C. Hojvat ${ }^{a}$, J.R. Hörandel ${ }^{80,81}$, P. Horvath ${ }^{33}$, M. Hrabovskýs ${ }^{33}$, T. Huege ${ }^{41,15}$, A. Insolia ${ }^{58,47}$, P.G. Isar ${ }^{74}$, P. Janecek ${ }^{32}$, J.A. Johnsen ${ }^{86}$, J. Jurysek ${ }^{32}$, A. Kääpä ${ }^{38}$, K.H. Kampert ${ }^{38}$, N. Karastathis ${ }^{41}$, B. Keilhauer ${ }^{41}$, J. Kemp ${ }^{42}$, A. Khakurdikar ${ }^{80}$, V.V. Kizakke Covilakam ${ }^{8,41}$, H.O. Klages ${ }^{41}$, M. Kleifges ${ }^{40}$, J. Kleinfeller ${ }^{10}$, M. Köpke ${ }^{39}$, N. Kunka ${ }^{40}$, B.L. Lago ${ }^{17}$, R.G. Lang ${ }^{20}$, N. Langner ${ }^{42}$, M.A. Leigui de Oliveira ${ }^{24}$, V. Lenok ${ }^{41}$, A. Letessier-Selvon ${ }^{35}$, I. Lhenry-Yvon ${ }^{34}$, D. Lo Presti ${ }^{58,47}$, L. Lopes ${ }^{72}$, R. López ${ }^{64}$, L. Lu ${ }^{94}$, Q. Luce ${ }^{39}$, J.P. Lundquist ${ }^{76}$, A. Machado Payeras ${ }^{22}$, G. Mancarella ${ }^{56,48}$, D. Mandat ${ }^{32}$, B.C. Manning ${ }^{13}$, J. Manshanden ${ }^{43}$, P. Mantsch ${ }^{a}$, S. Marafico ${ }^{34}$, A.G. Mariazzi ${ }^{4}$, I.C. Mariş ${ }^{14}$, G. Marsella ${ }^{61,47}$, D. Martello ${ }^{56,48}$, S. Martinelli ${ }^{41,8}$, O. Martínez Bravo ${ }^{64}$, M. Mastrodicasa ${ }^{57,46}$, H.J. Mathes ${ }^{41}$, J. Matthews ${ }^{88}$, G. Matthiae ${ }^{62,51}$, E. Mayotte ${ }^{38}$, P.O. Mazur ${ }^{a}$, G. Medina-Tanco ${ }^{68}$, D. $\mathrm{Melo}^{8}$, A. Menshikov ${ }^{40}$, K.-D. Merenda ${ }^{86}$, S. Michal ${ }^{33}$, M.I. Micheletti ${ }^{6}$, L. Miramonti ${ }^{59,49}$, S. Mollerach ${ }^{1}$, F. Montanet ${ }^{36}$, C. Morello ${ }^{54,52}$, M. Mostafá ${ }^{91}$, A.L. Müller ${ }^{8}$, M.A. Muller ${ }^{22}$, K. Mulrey ${ }^{15}$, R. Mussa ${ }^{52}$, M. Muzio ${ }^{90}$, W.M. Namasaka ${ }^{38}$, A. Nasr-Esfahani ${ }^{38}$, L. Nellen ${ }^{68}$, M. Niculescu-Oglinzanu ${ }^{73}$, M. Niechciol ${ }^{44}$, D. Nitz ${ }^{89}$, D. Nosek ${ }^{31}$, V. Novotny ${ }^{31}$, L. Nožka ${ }^{33}$, A Nucita ${ }^{56,48}$, L.A. Núñez ${ }^{30}$, M. Palatka ${ }^{32}$, J. Pallotta ${ }^{2}$, P. Papenbreer ${ }^{38}$, G. Parente ${ }^{79}$, A. Parra ${ }^{64}$, J. Pawlowsky ${ }^{38}$, M. Pech ${ }^{32}$, F. Pedreira ${ }^{79}$, J. Pękala ${ }^{70}$, R. Pelayo ${ }^{65}$, J. Peña-Rodriguez ${ }^{30}$, E.E. Pereira Martins ${ }^{39,8}$, J. Perez Armand ${ }^{21}$, C. Pérez Bertolli ${ }^{8,41}$, M. Perlin ${ }^{8,41}$, L. Perrone $e^{56,48}$, S. Petrera ${ }^{45,46}$, T. Pierog ${ }^{41}$, M. Pimenta $^{72}$, V. Pirronello ${ }^{58,47}$, M. Platino ${ }^{8}$, B. Pont ${ }^{80}$, M. Pothast ${ }^{81,80}$, P. Privitera ${ }^{92}$, M. Prouza $^{32}$, A. Puyleart ${ }^{89}$, S. Querchfeld ${ }^{38}$, J. Rautenberg ${ }^{38}$, D. Ravignani ${ }^{8}$, M. Reininghaus ${ }^{41,8}$, J. Ridky ${ }^{32}$, F. Riehn ${ }^{72}$, M. Risse ${ }^{44}$, V. Rizi ${ }^{57,46}$, W. Rodrigues de Carvalho $^{21}$, J. Rodriguez Rojo ${ }^{11}$, M.J. Roncoroni ${ }^{8}$, S. Rossoni ${ }^{43}$, M. Roth ${ }^{41}$, E. Roulet ${ }^{1}$, A.C. Rovero ${ }^{5}$, P. Ruehl ${ }^{44}$, A. Saftoiu ${ }^{73}$, F. Salamida ${ }^{57,46}$, H. Salazar ${ }^{64}$, G. Salina ${ }^{51}$, J.D. Sanabria Gomez ${ }^{30}$, F. Sánchez ${ }^{8}$, E.M. Santos ${ }^{21}$, E. Santos ${ }^{32}$, F. Sarazin ${ }^{86}$, R. Sarmento ${ }^{72}$, C. Sarmiento-Cano ${ }^{8}$, R. Sato ${ }^{11}$, P. Savina ${ }^{56,48,34,94}$, 
C.M. Schäfer ${ }^{41}$, V. Scherini ${ }^{56,48}$, H. Schieler ${ }^{41}$, M. Schimassek ${ }^{39,8}$, M. Schimp ${ }^{38}$, F. Schlüter ${ }^{41,8}$, D. Schmidt ${ }^{39}$, O. Scholten ${ }^{84,15}$, P. Schovánek ${ }^{32}$, F.G. Schröder ${ }^{93,41}$, S. Schröder ${ }^{38}$, J. Schulte ${ }^{42}$, S.J. Sciutto ${ }^{4}$, M. Scornavacche ${ }^{8,41}$, A. Segreto ${ }^{53,47}$, S. Sehgal ${ }^{38}$, R.C. Shellard ${ }^{16}$, G. Sigl ${ }^{43}$, G. Silli ${ }^{8,41}$, O. Sima ${ }^{73, f}$, R. Šmída ${ }^{92}$, P. Sommers ${ }^{91}$, J.F. Soriano ${ }^{87}$, J. Souchard ${ }^{36}$, R. Squartini ${ }^{10}$, M. Stadelmaier ${ }^{41,8}$, D. Stanca ${ }^{73}$, S. Stanič ${ }^{76}$, J. Stasielak ${ }^{70}$, P. Stassi ${ }^{36}$, A. Streich ${ }^{39,8}$, M. Suárez-Durán ${ }^{14}$, T. Sudholz ${ }^{13}$, T. Suomijärvi ${ }^{37}$, A.D. Supanitsky ${ }^{8}$, Z. Szadkowski ${ }^{71}$, A. Tapia ${ }^{29}$, C. Taricco ${ }^{63,52}$, C. Timmermans ${ }^{81,80}$, O. Tkachenko ${ }^{41}$, P. Tobiska ${ }^{32}$, C.J. Todero Peixoto ${ }^{19}$, B. Tomé ${ }^{2}$, Z. Torrès ${ }^{36}$, A. Travaini ${ }^{10}$, P. Travnicek $^{32}$, C. Trimarelli ${ }^{57,46}$, M. Tueros ${ }^{4}$, R. Ulrich ${ }^{41}$, M. Unger ${ }^{41}$, L. Vaclavek ${ }^{33}$, M. Vacula ${ }^{33}$, J.F. Valdés Galicia ${ }^{68}$, L. Valore ${ }^{60,50}$, E. Varela ${ }^{64}$, A. Vásquez-Ramírez ${ }^{30}$, D. Veberič ${ }^{41}$, C. Ventura ${ }^{27}$, I.D. Vergara Quispe ${ }^{4}$, V. Verzi ${ }^{51}$, J. Vicha ${ }^{32}$, J. Vink ${ }^{83}$, S. Vorobiov ${ }^{76}$, H. Wahlberg ${ }^{4}$, C. Watanabe ${ }^{26}$, A.A. Watson ${ }^{c}$, M. Weber ${ }^{40}$, A. Weindl ${ }^{41}$, L. Wiencke ${ }^{86}$, H. Wilczyński ${ }^{70}$, M. Wirtz ${ }^{42}$, D. Wittkowski ${ }^{38}$, B. Wundheiler ${ }^{8}$, A. Yushkov $^{32}$, O. Zapparrata ${ }^{14}$, E. Zas ${ }^{79}$, D. Zavrtanik ${ }^{76,77}$, M. Zavrtanik ${ }^{77,76}$, L. Zehrer ${ }^{76}$

\footnotetext{
${ }^{1}$ Centro Atómico Bariloche and Instituto Balseiro (CNEA-UNCuyo-CONICET), San Carlos de Bariloche, Argentina

${ }^{2}$ Centro de Investigaciones en Láseres y Aplicaciones, CITEDEF and CONICET, Villa Martelli, Argentina

${ }^{3}$ Departamento de Física and Departamento de Ciencias de la Atmósfera y los Océanos, FCEyN, Universidad de Buenos Aires and CONICET, Buenos Aires, Argentina

${ }^{4}$ IFLP, Universidad Nacional de La Plata and CONICET, La Plata, Argentina

${ }^{5}$ Instituto de Astronomía y Física del Espacio (IAFE, CONICET-UBA), Buenos Aires, Argentina

${ }^{6}$ Instituto de Física de Rosario (IFIR) - CONICET/U.N.R. and Facultad de Ciencias Bioquímicas y Farmacéuticas U.N.R., Rosario, Argentina

${ }^{7}$ Instituto de Tecnologías en Detección y Astropartículas (CNEA, CONICET, UNSAM), and Universidad Tecnológica Nacional - Facultad Regional Mendoza (CONICET/CNEA), Mendoza, Argentina

${ }^{8}$ Instituto de Tecnologías en Detección y Astropartículas (CNEA, CONICET, UNSAM), Buenos Aires, Argentina

${ }^{9}$ International Center of Advanced Studies and Instituto de Ciencias Físicas, ECyT-UNSAM and CONICET, Campus Miguelete - San Martín, Buenos Aires, Argentina

${ }^{10}$ Observatorio Pierre Auger, Malargüe, Argentina

${ }^{11}$ Observatorio Pierre Auger and Comisión Nacional de Energía Atómica, Malargüe, Argentina

${ }^{12}$ Universidad Tecnológica Nacional - Facultad Regional Buenos Aires, Buenos Aires, Argentina

${ }^{13}$ University of Adelaide, Adelaide, S.A., Australia

${ }^{14}$ Université Libre de Bruxelles (ULB), Brussels, Belgium

${ }^{15}$ Vrije Universiteit Brussels, Brussels, Belgium

${ }^{16}$ Centro Brasileiro de Pesquisas Fisicas, Rio de Janeiro, RJ, Brazil

${ }^{17}$ Centro Federal de Educação Tecnológica Celso Suckow da Fonseca, Nova Friburgo, Brazil

${ }^{18}$ Instituto Federal de Educação, Ciência e Tecnologia do Rio de Janeiro (IFRJ), Brazil

${ }^{19}$ Universidade de São Paulo, Escola de Engenharia de Lorena, Lorena, SP, Brazil

${ }^{20}$ Universidade de São Paulo, Instituto de Física de São Carlos, São Carlos, SP, Brazil

${ }^{21}$ Universidade de São Paulo, Instituto de Física, São Paulo, SP, Brazil

${ }^{22}$ Universidade Estadual de Campinas, IFGW, Campinas, SP, Brazil

${ }^{23}$ Universidade Estadual de Feira de Santana, Feira de Santana, Brazil

${ }^{24}$ Universidade Federal do ABC, Santo André, SP, Brazil

${ }^{25}$ Universidade Federal do Paraná, Setor Palotina, Palotina, Brazil

${ }^{26}$ Universidade Federal do Rio de Janeiro, Instituto de Física, Rio de Janeiro, RJ, Brazil

${ }^{27}$ Universidade Federal do Rio de Janeiro (UFRJ), Observatório do Valongo, Rio de Janeiro, RJ, Brazil

${ }^{28}$ Universidade Federal Fluminense, EEIMVR, Volta Redonda, RJ, Brazil

${ }^{29}$ Universidad de Medellín, Medellín, Colombia

${ }^{30}$ Universidad Industrial de Santander, Bucaramanga, Colombia

${ }^{31}$ Charles University, Faculty of Mathematics and Physics, Institute of Particle and Nuclear Physics, Prague, Czech Republic

${ }^{32}$ Institute of Physics of the Czech Academy of Sciences, Prague, Czech Republic
} 
${ }^{33}$ Palacky University, RCPTM, Olomouc, Czech Republic

${ }^{34}$ CNRS/IN2P3, IJCLab, Université Paris-Saclay, Orsay, France

${ }^{35}$ Laboratoire de Physique Nucléaire et de Hautes Energies (LPNHE), Sorbonne Université, Université de Paris, CNRSIN2P3, Paris, France

${ }^{36}$ Univ. Grenoble Alpes, CNRS, Grenoble Institute of Engineering Univ. Grenoble Alpes, LPSC-IN2P3, 38000 Grenoble, France

${ }^{37}$ Université Paris-Saclay, CNRS/IN2P3, IJCLab, Orsay, France

${ }^{38}$ Bergische Universität Wuppertal, Department of Physics, Wuppertal, Germany

${ }^{39}$ Karlsruhe Institute of Technology (KIT), Institute for Experimental Particle Physics, Karlsruhe, Germany

${ }^{40}$ Karlsruhe Institute of Technology (KIT), Institut für Prozessdatenverarbeitung und Elektronik, Karlsruhe, Germany

${ }^{41}$ Karlsruhe Institute of Technology (KIT), Institute for Astroparticle Physics, Karlsruhe, Germany

${ }^{42}$ RWTH Aachen University, III. Physikalisches Institut A, Aachen, Germany

${ }^{43}$ Universität Hamburg, II. Institut für Theoretische Physik, Hamburg, Germany

${ }^{44}$ Universität Siegen, Department Physik - Experimentelle Teilchenphysik, Siegen, Germany

${ }^{45}$ Gran Sasso Science Institute, L'Aquila, Italy

${ }^{46}$ INFN Laboratori Nazionali del Gran Sasso, Assergi (L'Aquila), Italy

${ }^{47}$ INFN, Sezione di Catania, Catania, Italy

${ }^{48}$ INFN, Sezione di Lecce, Lecce, Italy

${ }^{49}$ INFN, Sezione di Milano, Milano, Italy

${ }^{50}$ INFN, Sezione di Napoli, Napoli, Italy

${ }^{51}$ INFN, Sezione di Roma "Tor Vergata", Roma, Italy

${ }^{52}$ INFN, Sezione di Torino, Torino, Italy

${ }^{53}$ Istituto di Astrofisica Spaziale e Fisica Cosmica di Palermo (INAF), Palermo, Italy

${ }^{54}$ Osservatorio Astrofisico di Torino (INAF), Torino, Italy

${ }^{55}$ Politecnico di Milano, Dipartimento di Scienze e Tecnologie Aerospaziali , Milano, Italy

${ }^{56}$ Università del Salento, Dipartimento di Matematica e Fisica "E. De Giorgi”, Lecce, Italy

${ }^{57}$ Università dell'Aquila, Dipartimento di Scienze Fisiche e Chimiche, L'Aquila, Italy

${ }^{58}$ Università di Catania, Dipartimento di Fisica e Astronomia, Catania, Italy

${ }^{59}$ Università di Milano, Dipartimento di Fisica, Milano, Italy

${ }^{60}$ Università di Napoli "Federico II", Dipartimento di Fisica "Ettore Pancini”, Napoli, Italy

${ }^{61}$ Università di Palermo, Dipartimento di Fisica e Chimica "E. Segrè”, Palermo, Italy

${ }^{62}$ Università di Roma "Tor Vergata", Dipartimento di Fisica, Roma, Italy

${ }^{63}$ Università Torino, Dipartimento di Fisica, Torino, Italy

${ }^{64}$ Benemérita Universidad Autónoma de Puebla, Puebla, México

${ }^{65}$ Unidad Profesional Interdisciplinaria en Ingeniería y Tecnologías Avanzadas del Instituto Politécnico Nacional (UPIITA-IPN), México, D.F., México

${ }^{66}$ Universidad Autónoma de Chiapas, Tuxtla Gutiérrez, Chiapas, México

${ }^{67}$ Universidad Michoacana de San Nicolás de Hidalgo, Morelia, Michoacán, México

${ }^{68}$ Universidad Nacional Autónoma de México, México, D.F., México

${ }^{69}$ Universidad Nacional de San Agustin de Arequipa, Facultad de Ciencias Naturales y Formales, Arequipa, Peru

${ }^{70}$ Institute of Nuclear Physics PAN, Krakow, Poland

${ }^{71}$ University of Łódź, Faculty of High-Energy Astrophysics, Łódź, Poland

${ }^{72}$ Laboratório de Instrumentação e Física Experimental de Partículas - LIP and Instituto Superior Técnico - IST, Universidade de Lisboa - UL, Lisboa, Portugal

73 "Horia Hulubei” National Institute for Physics and Nuclear Engineering, Bucharest-Magurele, Romania

${ }^{74}$ Institute of Space Science, Bucharest-Magurele, Romania

${ }^{75}$ University Politehnica of Bucharest, Bucharest, Romania

${ }^{76}$ Center for Astrophysics and Cosmology (CAC), University of Nova Gorica, Nova Gorica, Slovenia

${ }^{77}$ Experimental Particle Physics Department, J. Stefan Institute, Ljubljana, Slovenia

${ }^{78}$ Universidad de Granada and C.A.F.P.E., Granada, Spain

${ }^{79}$ Instituto Galego de Física de Altas Enerxías (IGFAE), Universidade de Santiago de Compostela, Santiago de Compostela, Spain 
${ }^{80}$ IMAPP, Radboud University Nijmegen, Nijmegen, The Netherlands

${ }^{81}$ Nationaal Instituut voor Kernfysica en Hoge Energie Fysica (NIKHEF), Science Park, Amsterdam, The Netherlands

${ }^{82}$ Stichting Astronomisch Onderzoek in Nederland (ASTRON), Dwingeloo, The Netherlands

${ }^{83}$ Universiteit van Amsterdam, Faculty of Science, Amsterdam, The Netherlands

${ }^{84}$ University of Groningen, Kapteyn Astronomical Institute, Groningen, The Netherlands

${ }^{85}$ Case Western Reserve University, Cleveland, OH, USA

${ }^{86}$ Colorado School of Mines, Golden, CO, USA

${ }^{87}$ Department of Physics and Astronomy, Lehman College, City University of New York, Bronx, NY, USA

${ }^{88}$ Louisiana State University, Baton Rouge, LA, USA

${ }^{89}$ Michigan Technological University, Houghton, MI, USA

${ }^{90}$ New York University, New York, NY, USA

${ }^{91}$ Pennsylvania State University, University Park, PA, USA

${ }^{92}$ University of Chicago, Enrico Fermi Institute, Chicago, IL, USA

${ }^{93}$ University of Delaware, Department of Physics and Astronomy, Bartol Research Institute, Newark, DE, USA

${ }^{94}$ University of Wisconsin-Madison, Department of Physics and WIPAC, Madison, WI, USA

${ }^{a}$ Fermi National Accelerator Laboratory, Fermilab, Batavia, IL, USA

${ }^{b}$ Max-Planck-Institut für Radioastronomie, Bonn, Germany

${ }^{c}$ School of Physics and Astronomy, University of Leeds, Leeds, United Kingdom

${ }^{d}$ Colorado State University, Fort Collins, CO, USA

${ }^{e}$ now at Hakubi Center for Advanced Research and Graduate School of Science, Kyoto University, Kyoto, Japan

${ }^{f}$ also at University of Bucharest, Physics Department, Bucharest, Romania 